\title{
Accuracy of computer-aided design models of the jaws produced using ultra-low MDCT doses and ASIR and MBIR
}

\author{
Asma'a A. Al-Ekrish ${ }^{1}$ (D) Sara A. Alfadda ${ }^{2}$. Wadea Ameen ${ }^{3} \cdot$ Romed Hörmann ${ }^{4}$. Wolfgang Puelacher ${ }^{5}$. \\ Gerlig Widmann ${ }^{6}$
}

Received: 3 January 2018 / Accepted: 6 June 2018 / Published online: 16 June 2018

(c) The Author(s) 2018

\begin{abstract}
Purpose To compare the surface of computer-aided design (CAD) models of the maxilla produced using ultra-low MDCT doses combined with filtered backprojection (FBP), adaptive statistical iterative reconstruction (ASIR) and model-based iterative reconstruction (MBIR) reconstruction techniques with that produced from a standard dose/FBP protocol.

Methods A cadaveric completely edentulous maxilla was imaged using a standard dose protocol (CTDIvol: $29.4 \mathrm{mGy}$ ) and FBP, in addition to 5 low dose test protocols (LD1-5) (CTDIvol: 4.19, 2.64, 0.99, 0.53, and 0.29 mGy) reconstructed with FBP, ASIR 50, ASIR 100, and MBIR. A CAD model from each test protocol was superimposed onto the reference model using the 'Best Fit Alignment' function. Differences between the test and reference models were analyzed as maximum and mean deviations, and root-mean-square of the deviations, and color-coded models were obtained which demonstrated the location, magnitude and direction of the deviations.

Results Based upon the magnitude, size, and distribution of areas of deviations, CAD models from the following protocols were comparable to the reference model: FBP/LD1; ASIR 50/LD1 and LD2; ASIR 100/LD1, LD2, and LD3; MBIR/LD1. The following protocols demonstrated deviations mostly between $1-2 \mathrm{~mm}$ or under $1 \mathrm{~mm}$ but over large areas, and so their effect on surgical guide accuracy is questionable: FBP/LD2; MBIR/LD2, LD3, LD4, and LD5. The following protocols demonstrated large deviations over large areas and therefore were not comparable to the reference model: FBP/LD3, LD4, and LD5; ASIR 50/LD3, LD4, and LD5; ASIR 100/LD4, and LD5.

Conclusions When MDCT is used for CAD models of the jaws, dose reductions of $86 \%$ may be possible with FBP, $91 \%$ with ASIR 50, and 97\% with ASIR 100. Analysis of the stability and accuracy of CAD/CAM surgical guides as directly related to the jaws is needed to confirm the results.
\end{abstract}

Keywords Multidetector computed tomography $\cdot$ Computer-aided surgery $\cdot$ Radiography, Dental, Digital $\cdot$ Image-guided surgery $\cdot$ Radiation dosage

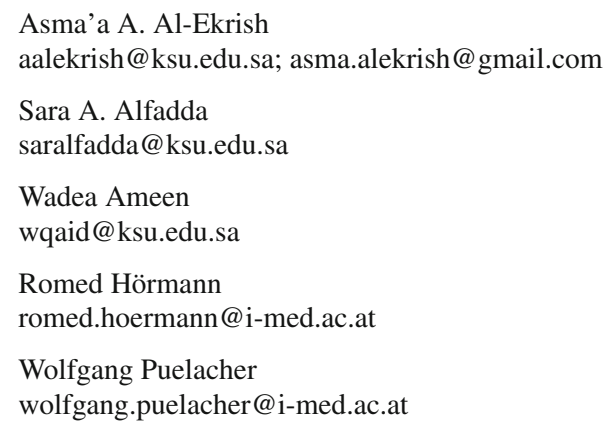

Gerlig Widmann

gerlig.widmann@i-med.ac.at

1 Department of Oral Medicine and Diagnostic Sciences, College of Dentistry, King Saud University, 60169, Riyadh 11545, Saudi Arabia

2 Department of Prosthetic Dental Sciences, College of Dentistry, King Saud University, 60169, Riyadh 11545, Saudi Arabia

3 Department of Industrial Engineering, Advanced Manufacturing Institute, College of Engineering, King Saud University, 800, Riyadh 11421, Saudi Arabia 


\section{Introduction}

Multidetector computed tomography (MDCT) is considered one of the most accurate modalities in production of computer-aided design (CAD) models of the jaws. The accuracy of $3 \mathrm{D}$ model production is important when MDCT images are used for implant planning with subsequent production of surgical guides for dental implant surgery. However, the increasing use of MDCT is considered one of the causes for the increasing collective dose of ionizing radiation to populations [1]; therefore, MDCT protocols with reduced or lower doses and which do not adversely affect diagnostic accuracy should be used whenever possible [2,3].

Using MDCT images produced with an $88 \%$ reduction in dose (compared to a standard clinical protocol), Loubel et al. [4] obtained accurate CAD models of the jaws. However, further reductions in dose may be limited by the increasing imaging noise [3,5] which may theoretically affect thresholding and image segmentation accuracy. The iterative reconstruction techniques (IRTs) of Adaptive Statistical Iterative Reconstruction (ASIR) and Model-Based Iterative Reconstruction (MBIR) have allowed the use of ultra-low MDCT doses with reduced noise levels, as compared with the traditionally used filtered backprojection technique (FBP) $[6,7]$. Such noise reduction may potentially improve the thresholding of the MDCT datasets.

When comparing positional accuracy of the jaws following orthognathic surgery, a root-mean-square of the difference (RMSD) of $0.5 \mathrm{~mm}$ between CAD models of the planned surgery and actual jaw position is considered to be clinical acceptable [8]. However, we are unaware of any recommended acceptable value for deviations in CAD models when they are used for construction of surgical guides for dental implant surgery. At the time of writing, to our knowledge, there is no information in the published literature regarding the effect of ultra-low MDCT doses in combination with various IRTs on the accuracy of CAD models of the jaws. The effect on accuracy of CAD models needs to be investigated to understand how the use of ultra-low dose IRT protocols may influence the accuracy of CAD-/CAMproduced surgical guides. The information may aid in dose optimization of MDCT in oral implant imaging. Therefore, this study aimed to compare the surface of CAD models of the maxilla produced using ultra-low doses combined with

4 Division of Clinical and Functional Anatomy, Medical University of Innsbruck, Müllerstrasse 59, 6020 Innsbruck, Austria

5 Department of CMF Surgery, Medical University of Innsbruck, Anichstr. 35, 6020 Innsbruck, Austria

6 Department of Radiology, Medical University of Innsbruck, Anichstr. 35, 6020 Innsbruck, Austria
FBP, ASIR and MBIR with the surface of a model produced from a standard dose/FBP protocol.

\section{Materials and methods}

\section{Cadaver selection}

A cadaveric head with a completely edentulous maxilla was used in the study. The head was part of a body donated by a person who had given his informed consent for its use for scientific and educational purposes prior to death and the study fulfilled all requirements necessary for studies on human cadavers according to the regulations of the Division of Clinical and Functional Anatomy, Medical University of Innsbruck $[9,10]$.

\section{Imaging}

The entire skull was scanned using a 64-multi-slice CT scanner (Discovery CT750 HD, GE Healthcare, Vienna, Austria) using a standard dose reference protocol and five low dose test protocols. The low dose test protocols were obtained by altering various exposure parameters in order to study the influence of each parameter on the results. The volume CT dose index $\left(\mathrm{CTDI}_{v o l}\right)$ for each protocol was obtained from the digital imaging and communication in medicine (DICOM) tags (Table 1). The reference dose protocol was reconstructed with FBP, and each low dose test protocol (TP) was reconstructed with FBP in addition to ASIR-50, ASIR-100, and MBIR. Bone convolution kernel was used except with MBIR, for which only standard convolution kernel was available. The slice thickness used in all protocols was $0.625 \mathrm{~mm}$. The matrix size was $512 \times 512$, with a pixel size of 0.391 , for the FBP and ASIR protocols. For the MBIR protocols, the matrix size was $1024 \times 1024$, with a pixel size of 0.195 .

\section{Production of CAD models}

The MDCT DICOM datasets were imported into Mimics software, version 10.01 (Materialise, Leuven, Belgium), which has been demonstrated to produce models of high accuracy [11], and the maxilla from each MDCT dataset was segmented in the following way:

1. The preset program in the MIMICS software for segmentation of "Bone" was selected. The Hounsfield Units (HU) threshold levels for this preset were 226-3071 HU.

2. The thresholding mask was cropped to include only the axial sections containing the maxilla from a level 6 slices above palate to several sections inferior to the crest of the maxillary alveolar ridge. 
Table 1 Exposure parameters used with the reference and test protocols (CTDIvol: volume CT Dose Index)

\begin{tabular}{lrrlll}
\hline Exposure protocol & \multicolumn{1}{c}{$\mathrm{kV}$} & $\mathrm{mA}$ & Rotation time (s) & Pitch & CTDI vol (mGy) \\
\hline Reference & 120 & 100 & 1.0 & 0.53125 & 29.4 \\
Test protocol 1 & 100 & 35 & 0.5 & 0.53125 & 4.19 \\
Test protocol 2 & 80 & 40 & 0.5 & 0.53125 & 2.64 \\
Test protocol 3 & 80 & 15 & 0.5 & 0.53125 & 0.99 \\
Test protocol 4 & 80 & 10 & 0.4 & 0.53125 & 0.53 \\
Test protocol 5 & 80 & 10 & 0.4 & 0.96875 & 0.29
\end{tabular}

3. Region growing of the mask was performed from the anterior maxillary ridge which led to exclusion of all floating pixels and structures other than the maxilla.

Each CAD model was then converted to a binary stereolithography (STL) file by use of the "Optimal" quality setting.

\section{Comparison of CAD model geometry}

The STL files were imported into the Geomagic Qualify 2013 software (3D systems, Rock Hill, South Carolina, USA), and each of the 20 test models was superimposed onto the reference model using the Best Fit Alignment function. The discrepancy between the surfaces of the CAD models were analyzed by comparing the position of 1500 points on the test model relative to their position on the reference model. The differences were analyzed as positive and negative deviations. A positive deviation indicated the test model was larger than the reference model on the outer surface of the maxilla, and a negative deviation indicated the test model was smaller (Fig. 1). The maximum and mean positive and negative deviations between each test model and the reference model were calculated, as well as the root-mean-square of the deviations (RMSD). The superimposition analyses were repeated three times, and the average values of the deviations and the RMSD obtained by the three analyses were calculated. All superimposition analyses were performed by an experienced operator blinded to the exposure and reconstruction protocols used to produce the test models. The reliability of the software in calculation of the deviations was tested by calculation of the intraclass correlation coefficient (ICC) for the mean, maximum, standard deviation, and RMS of the deviations obtained from the three superimposition analyses [using SPSS version 24 (IBM, Armonk, New York, United States)]. For each superimposition, a color-coded model was also obtained which demonstrated the location, magnitude and direction of the discrepancies between the reference and test models using a color-coded scale (Fig. 2). A descriptive analysis was then performed to analyze the position of the areas with deviations and their severity. Potential suitability of the test CAD models for the production of a surgical guide for dental implant guided surgery were evaluated by an experienced prosthodontist and radiologist based upon the magnitude, size of area, and distribution of areas which showed deviations larger than $0.5 \mathrm{~mm}$ at the following sites (in order of significance in terms of implant placement accuracy): crest of the residual ridge, buccal slope of the ridge, palatal slope of the ridge, and palate.

\section{Results}

Electronic supplementary material 1 shows the CAD models obtained by the reference and test protocols. Superimposition analyses between the test and reference models demonstrated that the pattern of distribution and magnitude of the deviations varied according to reconstruction technique and dose protocol (Electronic supplementary material 2). The ICC of the mean and maximum deviations and the RMSD obtained by the repeated analyses were 1.0, 0.995, and 1.0, respectively (Sig. level $<0.001$ ).

Table 2 demonstrates the magnitude of the deviations between the reference and test models. Decreasing dose led to increasing magnitude and larger areas of discrepancies with all the reconstruction techniques. However, MBIR showed less variability with dose reductions compared with the other reconstruction techniques. The maximum and mean deviations and the RMSD between the test models and the reference model increased with each dose reduction with all the reconstruction techniques, but the increase in deviations was markedly less with MBIR (Table 2), and were also less with increasing ASIR percentage. The RMSD values for MBIR at all doses were less than $0.4 \mathrm{~mm}$. For each protocol, the mean positive and mean negative differences were nearly similar, indicating none of the protocols were associated with magnification or minification of the models.

The reconstruction technique associated with the largest magnitude and areas of deviations at any given dose protocol was FBP followed by ASIR 50 followed by ASIR 100 . The maximum deviations detected with FBP and ASIR were not localized to any particular region. The pattern of deviations in FBP and ASIR seemed influenced by the noise patterns in the MDCT datasets, appearing as discrete points 


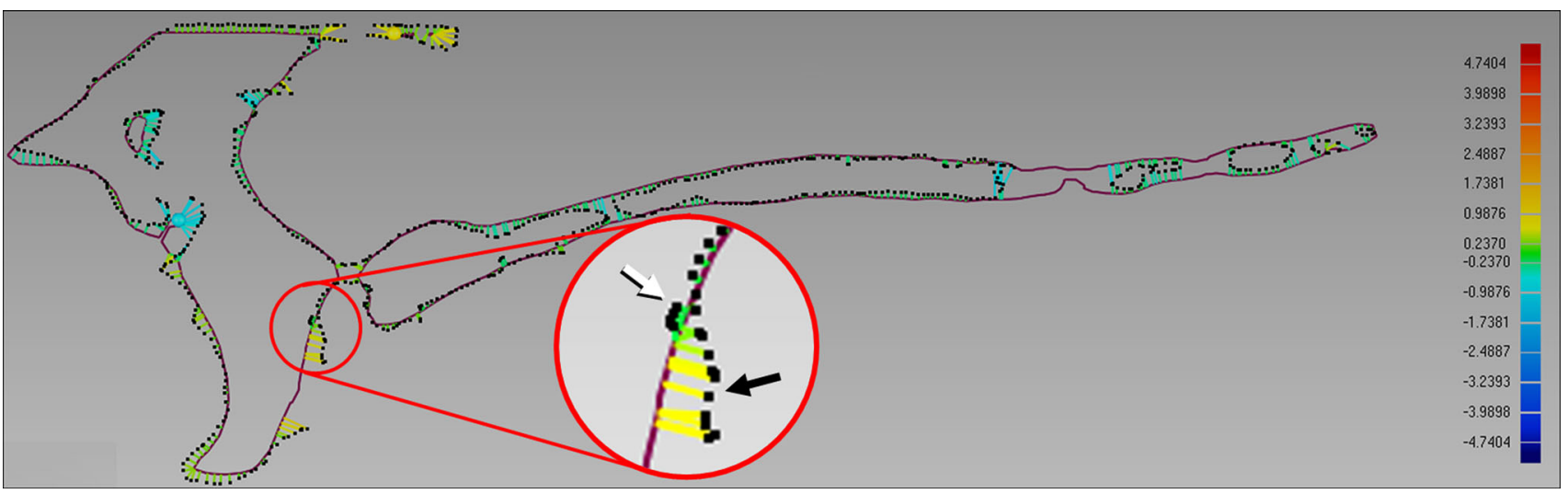

Fig. 1 Diagrammatic representation of sagittal section of superimposed models demonstrating magnitude of deviations as indicated by color scale; positive deviations represented by yellow-red bars (black arrow) and negative deviations represented by green-blue bars (white arrow). Yellow and blue circles indicate the maximum deviations

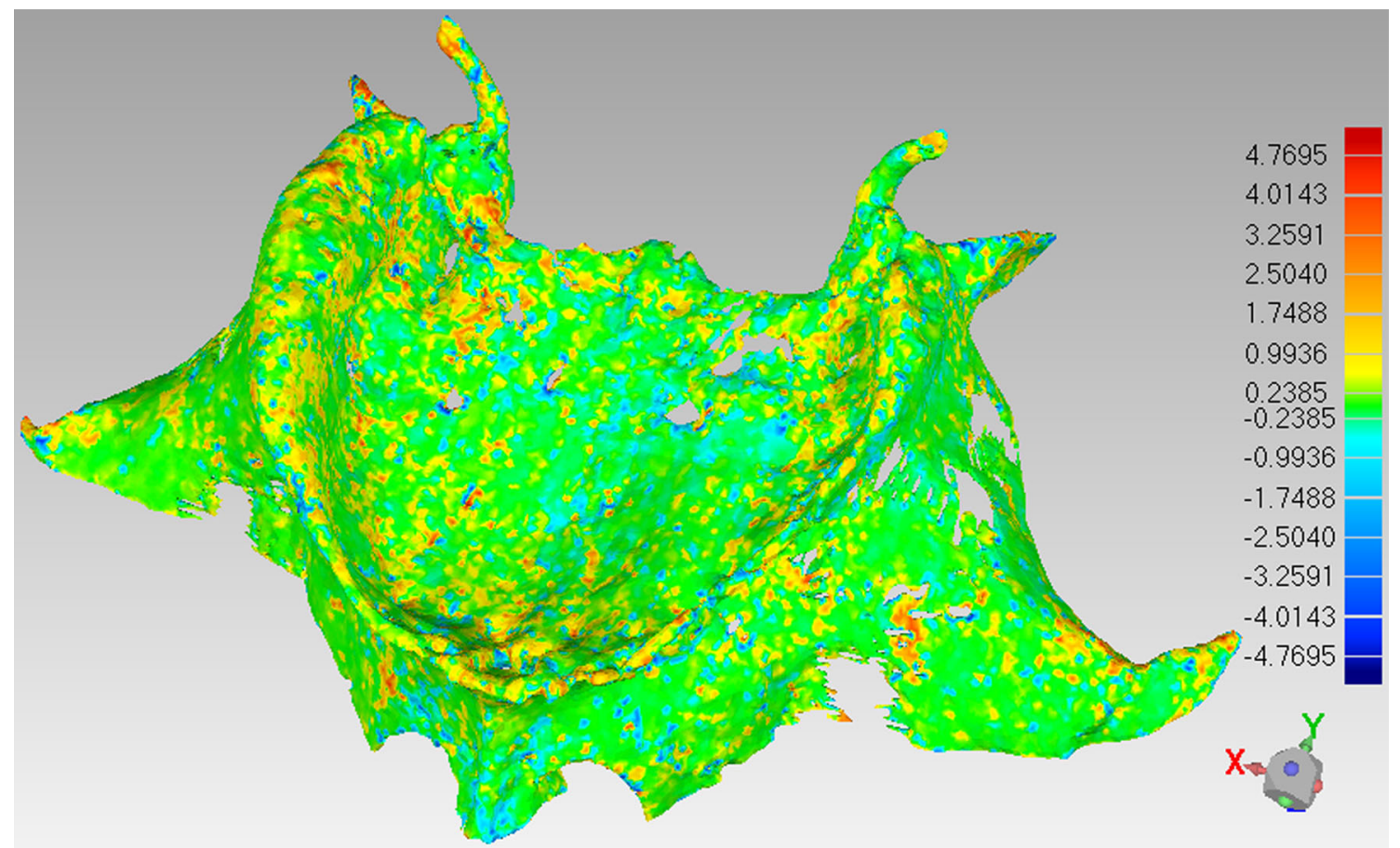

Fig. 2 Color-coded analysis demonstrating the location, magnitude and direction of the deviations (in $\mathrm{mm}$ ) between the reference model and a test model (FBP/Test protocol 4) (see color scale on the side)

of deviation coalesced together, with the amount and magnitude of the deviations increasing with increasing noise, which resulted from decreasing dose. With MBIR, the pattern of deviations did not appear related to noise in the MDCT data. The maximum deviations detected with MBIR were not localized to any particular region, and appeared at areas of over-smoothening of the surface of the model. The RMSD values obtained with MBIR were consistently the lowest of all the reconstruction techniques but the pattern of distribution of the deviations was different from the other techniques which precluded a direct comparison in this aspect.
With FBP, the model produced by the TP1 protocol demonstrated deviations mostly within $0.2 \mathrm{~mm}$, with a small number of points larger than $1 \mathrm{~mm}$ which were widely distributed and, thus, not expected to affect the accuracy of a resultant guide. The model produced by FBP and TP2 protocol demonstrated numerous deviation points greater than $1 \mathrm{~mm}$ coalesced together in the anterior palate and posterior aspect of the palate and right tuberosity region. Deviation points ranging in magnitude between $1-2 \mathrm{~mm}$ were situated on the crest and palatal slope of the ridge but more widely distributed. Therefore, the effect of the deviations on the 
Table 2 Numerical differences between each test model and the reference model expressed as Maximum (Max) and Mean deviations (positive and negative), standard deviation (SD) of the differences, and root of the mean square of the deviations (RMSD)

\begin{tabular}{|c|c|c|c|c|}
\hline & FBP & ASIR 50 & ASIR 100 & MBIR \\
\hline \multirow[t]{6}{*}{ Test protocol 1} & $\operatorname{Max}(+): 5.1$ & $\operatorname{Max}(+): 2.6$ & $\operatorname{Max}(+): 2.5$ & $\operatorname{Max}(+): 3.3$ \\
\hline & $\operatorname{Max}(-): 5.2$ & $\operatorname{Max}(-): 4.7$ & $\operatorname{Max}(-): 5.0$ & $\operatorname{Max}(-): 2.5$ \\
\hline & Mean (+): 0.2 & Mean (+): 0.2 & Mean (+): 0.1 & Mean (+): 0.1 \\
\hline & Mean (-): 0.2 & Mean (-): 0.1 & Mean (-): 0.1 & Mean (-): 0.1 \\
\hline & SD: 0.4 & SD: 0.3 & SD: 0.2 & SD: 0.2 \\
\hline & RMSD: 0.4 & RMSD: 0.3 & RMSD: 0.2 & RMSD: 0.2 \\
\hline \multirow[t]{6}{*}{ Test protocol 2} & $\operatorname{Max}(+): 6.2$ & $\operatorname{Max}(+): 5.2$ & $\operatorname{Max}(+): 4.8$ & $\operatorname{Max}(+): 4.4$ \\
\hline & $\operatorname{Max}(-): 5.9$ & $\operatorname{Max}(-): 5.9$ & $\operatorname{Max}(-): 3.4$ & $\operatorname{Max}(-): 3.0$ \\
\hline & Mean (+): 0.3 & Mean (+): 0.2 & Mean (+): 0.2 & Mean (+): 0.2 \\
\hline & Mean (-): 0.4 & Mean (-): 0.3 & Mean (-): 0.2 & Mean (-): 0.1 \\
\hline & SD: 0.6 & SD: 0.4 & SD: 0.3 & SD: 0.2 \\
\hline & RMSD: 0.6 & RMSD: 0.4 & RMSD: 0.3 & RMSD: 0.2 \\
\hline \multirow[t]{6}{*}{ Test protocol 3} & $\operatorname{Max}(+): 8.1$ & $\operatorname{Max}(+): 7.6$ & $\operatorname{Max}(+): 6.5$ & $\operatorname{Max}(+): 3.1$ \\
\hline & $\operatorname{Max}(-): 10.1$ & $\operatorname{Max}(-): 8.9$ & $\operatorname{Max}(-): 5.9$ & $\operatorname{Max}(-): 3.8$ \\
\hline & Mean (+): 0.7 & Mean (+): 0.4 & Mean (+): 0.3 & Mean (+): 0.2 \\
\hline & Mean (-): 1.0 & Mean (-): 0.5 & Mean (-): 0.3 & Mean (-): 0.2 \\
\hline & SD: 1.3 & SD: 0.8 & SD: 0.5 & SD: 0.3 \\
\hline & RMSD: 1.3 & RMSD: 0.8 & RMSD: 0.5 & RMSD: 0.3 \\
\hline \multirow[t]{6}{*}{ Test protocol 4} & $\operatorname{Max}(+): 6.8$ & $\operatorname{Max}(+): 7.0$ & $\operatorname{Max}(+): 6.0$ & $\operatorname{Max}(+): 3.7$ \\
\hline & $\operatorname{Max}(-): 10.4$ & $\operatorname{Max}(-): 9.0$ & $\operatorname{Max}(-): 6.4$ & $\operatorname{Max}(-): 3.6$ \\
\hline & Mean (+): 0.8 & Mean (+): 0.5 & Mean (+): 0.3 & Mean $(+): 0.3$ \\
\hline & Mean (-): 0.9 & Mean (-): 0.5 & Mean (-): 0.3 & Mean (-):0.2 \\
\hline & SD: 1.2 & SD: 0.8 & SD: 0.5 & SD: 0.3 \\
\hline & RMSD: 1.2 & RMSD: 0.8 & RMSD: 0.5 & RMSD: 0.3 \\
\hline \multirow[t]{6}{*}{ Test protocol 5} & $\begin{array}{l}\text { Analysis not performed due } \\
\text { to inability to segment the } \\
\text { maxilla. }\end{array}$ & $\operatorname{Max}(+): 13.4$ & $\operatorname{Max}(+): 6.2$ & $\operatorname{Max}(+): 4.6$ \\
\hline & & $\operatorname{Max}(-): 13.5$ & $\operatorname{Max}(-): 7.7$ & $\operatorname{Max}(-): 5.5$ \\
\hline & & Mean (+): 1.3 & Mean (+): 0.5 & Mean (+): 0.3 \\
\hline & & Mean (-): 1.4 & Mean (-): 0.6 & Mean (-): 0.3 \\
\hline & & SD: 2.1 & SD: 0.8 & SD: 0.4 \\
\hline & & RMSD: 2.1 & RMSD: 0.8 & RMSD: 0.4 \\
\hline
\end{tabular}

Numbers are the calculated average of three superimposition analyses. All units are in $\mathrm{mm}$ accuracy of a guide produced by FBP and TP 2 protocol is questionable, and may be elucidated by producing CAM surgical guides and testing their accuracy in implant surgeries. With FBP/TP 3 and TP 4, the magnitude of deviations and their distribution close to each other appear to preclude the production of surgical guides with the desired accuracy. With FBP and TP 5, a CAD model of the maxilla could not be segmented due to the large amount of surrounding noise. So a superimposition analysis for FBP/TP 5 was not performed because the comparison data (points for comparison with the surface of the reference model) were haphazardly oriented and too far away from the reference.

With ASIR 50/TP 1 protocol, the magnitude and distribution of the deviation points were less than that seen with FBP/TP 1. With ASIR 50/TP 2, the number of deviation points appeared greater in number and extent than seen with ASIR50/TP 1, but much less than those seen with FBP/TP 2. The magnitude of the deviations appeared within $1 \mathrm{~mm}$, with a small number of widely distributed negative deviation points larger than $1 \mathrm{~mm}$, which may possibly be clinically insignificant. Both ASIR50/TP 1 and TP 2 protocols may possibly facilitate the production of accurate surgical guides. For ASIR 50/TP 3, TP 4, and TP 5 protocols, the magnitude of deviations and their distribution close to each other appear to preclude the production of accurate surgical guides.

With ASIR 100/TP 1, very few areas showed negative deviations of approximately $1 \mathrm{~mm}$, and the ridge had almost no deviation points greater than $0.2 \mathrm{~mm}$. There was a questionable area at the zygomatic process of the maxilla which is not clinically significant in construction of a surgical guide. 
With ASIR 100/TP 2, the area and magnitude of deviations was less than FBP/TP 1, and the areas were widely distributed and small in size and mostly approximately positive $1 \mathrm{~mm}$. With ASIR 100/TP 3, most of the deviations were within $0.2 \mathrm{~mm}$. And, although there were numerous areas showing deviations at the ridge, the small number of areas with deviations larger than $1 \mathrm{~mm}$ were widely distributed which may possibly not affect the accuracy of a resultant surgical guide. Therefore, use of ASIR 100 combined with TP 1, TP 2, or TP 3 may possibly be useful in the production of surgical guides. For TP 4, widespread discrepancies approaching $1 \mathrm{~mm}$, with very few areas demonstrating larger deviations were considered to have a questionable effect on potential accuracy of a CAD/CAM guide. However, for ASIR 100/TP 5 the magnitude of deviations and their distribution close to each other appear to preclude the production of accurate surgical guides.

The areas of deviations seen with MBIR appeared as wide homogenous areas of positive deviations. MBIR/TP 1 showed wider areas of deviations compared with ASIR 50 and ASIR 100 at the same dose. The deviations were positive and within $0.5 \mathrm{~mm}$, and no areas of deviations were seen along the ridge. The large questionable area with missing bone in the CAD model was situated at the zygomatic process of the maxilla and so may possibly have no effect of on the accuracy of a surgical guide. Therefore, MBIR/TP 1 appears to be potentially suitable for use in construction of surgical guides. With MBIR/TP 2, wide areas of positive deviations within $0.6 \mathrm{~mm}$ were present which may possibly affect accuracy of a resultant surgical guide due to the large size of the affected areas and their locations on the crest and palatal slope of the ridge as well as the palate. With MBIR/TP 3 , larger areas of positive deviations were detected, as well as areas of negative deviations; MBIR/TP 4 and TP 5 showed larger areas and increasing degrees of deviations, along with areas of lack of bone at the crest of the ridge and buccal slope. The combined effect of extensive areas of small degree of deviations seen with MBIR/TP 2- TP 5 is questionable. The implications for guide production may be further elucidated after analysis of accuracy of CAD-/CAM-produced surgical guides in implant surgeries.

\section{Discussion}

The present study investigated the comparability of the surface of CAD models of an edentulous maxilla produced using ultra-low doses combined with FBP, ASIR and MBIR with the surface of a model produced from a standard dose/FBP protocol. The results indicated the following protocols produced comparable CAD models, and hence may potentially allow the production of accurate $\mathrm{CAD} / \mathrm{CAM}$ surgical guides: FBP/TP 1; ASIR 50/TP 1 and 2; ASIR 100/TP 1, 2, and 3; and MBIR/TP 1. Furthermore, the following protocols were found to have a questionable effect on CAD model accuracy which may be better determined by analysis of the accuracy of CAD/CAM surgical guides directly in implant surgeries: FBP/TP 2 and ASIR 100/TP 4. For the MBIR models, the accuracy of the models produced by MBIR/TP 2, 3 , 4 , and 5 was considered questionable because of the wide areas of approximately $0.5 \mathrm{~mm}$ deviations coalesced together and their positions relative to the jaw. Thus, although MBIR shows promise for the production of accurate CAD models at ultra-low doses, its ultimate effect on production of $\mathrm{CAD} / \mathrm{CAM}$ guides needs to be clarified by actual production of the guides and testing their accuracy during implant surgery. The pattern of deviations observed in the superimposition models suggests that the major cause of deviations in the FBP and ASIR models was the noise level, whereas for the MBIR models it may have been the effect of oversmoothening of the images.

For FBP and MBIR, reducing the $\mathrm{kV}$ in $\mathrm{TP} 2$ produced models of questionable usefulness for production of surgical guides, even though the $\mathrm{mA}$ was increased when compared to TP1. For ASIR 50 and ASIR 100, progressive reductions in $\mathrm{mA}$, with or without reductions in rotation time, produced models with questionable usefulness. However, the ultimate accuracy of CAD/CAM surgical guides depends on a multitude of factors in addition to accuracy of the CT images [12]. The present study, being a CAD model study, provides information on the isolated effect of the $\mathrm{CT}$ data on comparability of the models by eliminating the confounding factors of manufacturing and procedural errors. Furthermore, the tissue surface of surgical guides is best constructed based upon optical scans of stone casts or intraoral scans, and not directly from the CT scans. As such, the results of the present study should not be considered as evidence regarding the suitability of the test protocols for the production of CAD/CAM surgical guides. Rather, they may be used as an indicator which may direct CAD/CAM research toward specific protocols which show potential usefulness, thus reducing time and cost of investigating inappropriate protocols. As such, it is recommended that $\mathrm{CAD} / \mathrm{CAM}$ surgical guides be produced by the following protocols and tested for accuracy in implant surgery: FBP/TP 1 and 2; ASIR 50/TP 1 and 2; ASIR 100/TP 1, 2, 3, and 4; and MBIR with all the test protocols.

Some investigators consider an RMSD of $0.5 \mathrm{~mm}$ or less to be indicative of clinically acceptable accuracy of postoperative alignment compared to preoperative maxillofacial surgical simulations [8]. Based upon analysis of the magnitude and distributions of deviations obtained in the present study, almost all of the models considered to be potentially useful and recommended for further CAD/CAM studies have demonstrated an RMSD lower than 0.5. However, more CAD studies and further investigation of the accuracy of $\mathrm{CAD} / \mathrm{CAM}$ guides in implant surgery are necessary before 
RMSD values of CAD models can be correlated with clinically acceptable deviations in implant surgery.

The present study's finding of relative comparability of the FBP/TP2 model with the reference model is in agreement with the findings of Loubele et al. [4] who reported accurate segmentation of CAD models of a skull phantom with an MDCT CTDIvol similar to that imparted by TP 2 of the present study. In the present study, MBIR produced the smoothest CAD models and lowest deviations of all the reconstruction techniques; these findings are compatible with the findings of studies which found that MBIR demonstrated more marked improvements in image quality at low doses compared with other reconstruction techniques $[6,13]$.

The aggressive dose reductions investigated in the present study attempt to contribute to dose optimization of MDCT in implant imaging. With regards to imparted doses, ultra-low dose MDCT may have an advantage over CBCT when multiple implant sites need to be imaged. For the reported doses from ultra-low dose MDCT protocols [14] were found to be lower than those reported for several maxillofacial CBCT devices $[15,16]$. The accuracy of 3-D models printed from standard dose MDCT has been shown to be similar to or greater than that from CBCT [17,18], even though CBCT has been demonstrated to produce more accurate sectional images [19]. This advantage of MDCT over CBCT may be because of more accurate thresholding and segmentation demonstrated with MDCT [20].

In MDCT, one scan may be simultaneously reconstructed using various different convolution kernels. The obtained images may vary substantially depending on which kernel is chosen, e.g., high and low level bone kernels with smoother and sharper edges, or even soft tissue kernels. The potential of this advantage over CBCT in terms of segmentation accuracy is yet to be evaluated. For, the use of ultra-low doses and IRTs has been demonstrated in the present study to introduce deviations in the CAD models which may conceivably produce models of similar or lower accuracy than those produced by CBCT. In order to determine which modality and ultra-low dose MDCT protocol would be better suited for 3D model production, studies must be conducted comparing the accuracy of printed models and/or surgical guides produced from ultra-low dose MDCT with those from CBCT.

A limitation of the present study is that only a bone convolution kernel was used for FBP and ASIR. The bone kernel was used because sectional images obtained with a bone kernel reportedly demonstrated higher spatial resolution of images [21] and provided more accurate measurements of bone than those obtained with a standard convolution kernel [22]. And CAD/CAM production of surgical guides initially involves ridge measurements and implant simulations based upon sectional images. However, images reconstructed with standard kernels show less noise than bone kernels [23]. Therefore, it is conceivable that datasets pro- duced using standard kernel may have improved thresholding and segmentation accuracy. Development of reconstruction techniques and kernels which combine the advantages of a bone kernel (good spatial resolution) with the advantages of a standard kernel (low noise levels) may possibly allow similar dose reductions for both sectional images and CAD models used for implant imaging.

Another limitation of the study was that a completely edentulous maxilla was used, for some surgical guide manufacturers do not construct bone supported CAD/CAM guides for fully edentulous cases. The edentulous jaw was used in order to limit the studied effects of the test protocols on bone thresholding only. A maxilla was used as opposed to a mandible in order to provide wider areas of bone for comparison. However, the thin bone at some maxillary sites poses higher thresholding difficulties than the thick bone of the mandible and teeth. Thus, it is possible that accurate CAD models of the teeth and mandible may be obtained at lower doses than those possible for maxillary CAD models. Therefore, the implications of the results on CAD models used for tooth supported, tooth-tissue supported, and tissue supported surgical guides also need to be investigated. Further studies may be conducted using mandibles and jaws with tooth bounded and distal extension edentulous areas.

\section{Conclusions}

When MDCT is used for CAD of the jaws, dose reductions of $86 \%$ may be possible with FBP, $91 \%$ with ASIR50, and 97\% with ASIR100. The powerful effect of noise reduction with MBIR on the potential accuracy of surgical guides could not be determined using CAD models. Analysis of the stability and accuracy of CAD/CAM surgical guides as directly related to the jaws in implant surgery is needed to confirm the results obtained with FBP and ASIR and to clarify the effect of MBIR and ultra-low doses.

Acknowledgements The authors extend their appreciation to the Deanship of Scientific Research at King Saud University for funding this work through research group number RGP-1438-037. The authors wish to thank Professor Abdulrahman M. Al-Ahmari, Dean of the Advanced Manufacturing Institute, King Saud University, for his aid in facilitation of the study. The authors also thank individuals who donated their bodies and tissues for the advancement of education and research. All authors declare no conflict of interest.

\section{Compliance with ethical standards}

Conflict of interest All authors declare that they have no conflict of interest.

Ethical approval Institutional Review Board approval was not required because the bodies used in the study were donated by people who had 
given their informed consent for their use for scientific and educational purposes prior to death, and the study fulfilled all requirements necessary for studies on human cadavers according to the regulations of the Division of Clinical and Functional Anatomy, Medical University of Innsbruck.

Funding The authors extend their appreciation to the Deanship of Scientific Research at King Saud University for funding this work through research group number RGP-1438-037.

Open Access This article is distributed under the terms of the Creative Commons Attribution 4.0 International License (http://creativecomm ons.org/licenses/by/4.0/), which permits unrestricted use, distribution, and reproduction in any medium, provided you give appropriate credit to the original author(s) and the source, provide a link to the Creative Commons license, and indicate if changes were made.

\section{References}

1. United Nations Scientific Committee on the Effects of Atomic Radiation U Sources and effects of ionizing radiation. In: Official records of the general assembly, 63rd session, Supplement No. 46., New York, 2010-2008. United Nations

2. Tyndall DA, Price JB, Tetradis S, Ganz SD, Hildebolt C, Scarfe WC (2012) Position statement of the American Academy of Oral and Maxillofacial Radiology on selection criteria for the use of radiology in dental implantology with emphasis on cone beam computed tomography. Oral Surg Oral Med Oral Pathol Oral Radiol 113(6):817-826

3. Kalra MK, Maher MM, Toth TL, Hamberg LM, Blake MA, Shepard JA, Saini S (2004) Strategies for CT radiation dose optimization. Radiology 230(3):619-628. https://doi.org/10.1148/ radiol.2303021726

4. Loubele M, Jacobs R, Maes F, Schutyser F, Debaveye D, Bogaerts R, Coudyzer W, Vandermeulen D, Van Cleynenbreugel J, Marchal G (2005) Radiation dose vs. image quality for low-dose CT protocols of the head for maxillofacial surgery and oral implant planning. Radiat Prot Dosimetry 117(1-3):211-216

5. McCollough CH, Primak AN, Braun N, Kofler J, Yu L, Christner J (2009) Strategies for reducing radiation dose in CT. Radiol Clin North Am 47(1):27-40. https://doi.org/10.1016/j.rcl.2008.10.006

6. Widmann G, Al-Shawaf R, Schullian P, Al-Sadhan R, Hormann R, Al-Ekrish AA (2017) Effect of ultra-low doses, ASIR and MBIR on density and noise levels of MDCT images of dental implant sites. Eur Radiol 27(5):2225-2234. https://doi.org/10. 1007/s00330-016-4588-8

7. Widmann G, Bischel A, Stratis A, Bosmans H, Jacobs R, Gassner EM, Puelacher W, Pauwels R (2017) Spatial and contrast resolution of ultralow dose dentomaxillofacial CT imaging using iterative reconstruction technology. Dentomaxillofac Radiol 46(4):20160452. https://doi.org/10.1259/dmfr.20160452

8. Lin H-H, Chang H-W, Lo L-J (2015) Development of customized positioning guides using computer-aided design and manufacturing technology for orthognathic surgery. IJCARS 10(12):2021-2033

9. McHanwell S, Brenner E, Chirculescu A, Drukker J, van Mameren H, Mazzotti G, Pais D, Paulsen F, Plaisant O, Caillaud M, Laforêt E, Riederer B, Sanudo J, Bueno-Lopez J, Donate F, Sprumont P, Teofilovski-Parapid G, Moxham B (2008) The legal and ethical framework governing Body Donation in Europe- a review of current practice and recommendations for good practice. Eur J Anat 12(1): $1-24$

10. Riederer B, Bolt S, Brenner E, Bueno-Lopez J, Cirulescu A, Davies D, De Caro R, Gerrits P, McHanwell S, Pais D, Paulsen F, Plaisant O, Sendemir E, Stabile I, Moxham B (2012) The legal and ethical framework governing Body Donation in Europe-1st update on current practice. Eur J Anat 16(1):1-21

11. Nizam A, Gopal R, Naing L, Hakim A, Samsudin A (2006) Dimensional accuracy of the skull models produced by rapid prototyping technology using stereolithography apparatus. Arch Orofac Sci 1:60-66

12. Vercruyssen M, Jacobs R, Van Assche N, van Steenberghe D (2008) The use of CT scan based planning for oral rehabilitation by means of implants and its transfer to the surgical field: a critical review on accuracy. J Oral Rehabil 35(6):454-474. https://doi.org/10.1111/ j.1365-2842.2007.01816.x

13. Widmann G, Bischel A, Stratis A, Kakar A, Bosmans H, Jacobs R, Gassner EM, Puelacher W, Pauwels R (2016) Ultralow dose dentomaxillofacial CT imaging and iterative reconstruction techniques: variability of Hounsfield units and contrast-to-noise ratio. Br J Radiol 89(1060):20151055. https://doi.org/10.1259/bjr. 20151055

14. Widmann G, Dalla Torre D, Hoermann R, Schullian P, Gassner EM, Bale R, Puelacher W (2015) Ultralow-dose computed tomography imaging for surgery of midfacial and orbital fractures using ASIR and MBIR. Int J Oral Maxillofac Surg 44(4):441-446. https://doi. org/10.1016/j.ijom.2015.01.011

15. Ludlow J, Timothy R, Walker C, Hunter R, Benavides E, Samuelson D, Scheske M (2015) Effective dose of dental CBCT-a meta analysis of published data and additional data for nine CBCT units. Dentomaxillofac Radiol 44(1):20140197

16. Kyriakou Y, Kolditz D, Langner O, Krause J, Kalender W (2011) Digital volume tomography (DVT) and multislice spiral CT (MSCT): an objective examination of dose and image quality. Rofo 183(2):144-153. https://doi.org/10.1055/s-0029-1245709

17. Primo B, Presotto A, de Oliveira H, Gassen H, Miguens S, Silva A, Hernandez P (2012) Accuracy assessment of prototypes produced using multi-slice and cone-beam computed tomography. Int J Oral Maxillofac Surg 41:1291-1295

18. Liang X, Lambrichts I, Sun Y, Denis K, Hassan B, Li L, Pauwels R, Jacobs R (2010) A comparative evaluation of cone beam computed tomography (CBCT) and multi-slice CT (MSCT). Part II: On 3D model accuracy. Eur J Radiol 75(2):270-274

19. Al-Ekrish A, Ekram M (2011) A comparative study of the accuracy and reliability of multidetector computed tomography and cone beam computed tomography in the assessment of dental implant site dimensions. Dentomaxillofac Radiol 40(2):67-75

20. Mah P, Reeves TE, McDavid WD (2010) Deriving Hounsfield units using grey levels in cone beam computed tomography. Dentomaxillofac Radiol 39(6):323-335. https://doi.org/10.1259/dmfr/ 19603304

21. Blendl C, Fiebich M, Voigt J, Selbach M, Uphoff C (2012) [Investigation on the $3 \mathrm{D}$ geometric accuracy and on the image quality (MTF, SNR and NPS) of volume tomography units (CT, CBCT and DVT)]. RoFo. Fortschritte auf dem Gebiete der Rontgenstrahlen und der Nuklearmedizin 184(1):24-31

22. Loubele M, Van Assche N, Carpentier K, Maes F, Jacobs R, van Steenberghe D, Suetens P (2008) Comparative localized linear accuracy of small-field cone-beam CT and multislice CT for alveolar bone measurements. Oral Surg Oral Med Oral Pathol Oral Radiol Endod 105(4):512-518. https://doi.org/10.1016/j.tripleo. 2007.05.004

23. Hoxworth JM, Lal D, Fletcher GP, Patel AC, He M, Paden RG, Hara AK (2014) Radiation dose reduction in paranasal sinus CT using model-based iterative reconstruction. AJNR Am J Neuroradiol 35(4):644-649. https://doi.org/10.3174/ajnr.A3749 